International Journal of Mathematics Research.

ISSN 0976-5840 Volume 12, Number 1 (2020), pp. 1-4

(C) International Research Publication House

https://dx.doi.org/10.37624/IJMR/12.1.2020.1-4

\title{
Directed Strongly Regular Graphs and their Codes: Determinant Factorization for a Tight $p$-rank
}

\author{
Amadou Keita \\ Department of Mathematics, University of The Gambia, Brikama Campus, \\ P.O. Box 3530, Serrekunda, The Gambia.
}

\begin{abstract}
The elementary divisors of the adjacency matrix $A$ of a directed strongly regular graph are analysed to establish a tight bound for the rank over a finite field using the determinant factorization.
\end{abstract}

Keywords and phrases: Determinant, adjacency matrix, $p$-rank, directed strongly regular graphs, eigenvalues.

\section{INTRODUCTION}

This paper seeks to address the main open problem given in [1]. The open problem was trying to establish a tight bound for the rank of an adjacency matrix of a directed strongly regular graph over a finite field, which is equivalent to finding the dimension of the codes. The statement was conjectured in [1] as follows:

Conjecture 1. [1] If $p^{j} \operatorname{divides} \operatorname{det}(A) \neq 0$ exactly, then the rank of $A$ over $\mathbb{F}_{p}$ is $v-j$.

The conjecture was a hasty one and so there exists some counterexamples to it. The conjecture is modified and the modified form is recorded as Theorem 1 and a proof for it is given.

In this paper, we give a short background in Section 2, discuss some counterexamples in Section 3, give our results in Section 4 and then conclude in Section 5. 


\section{BACKGROUND}

Suppose $G$ is a directed graph on $v$ vertices. Let $A$ be a matrix of size $v$ by $v$ with entries from the set $\{0,1\}$. Then $A$ is the adjacency matrix of a directed strongly regular graph, if, for parameters $(v, k, t, \lambda, \mu), 0<t<k$, and satisfies

$$
\begin{aligned}
& \star A J=J A=k J \\
& \star A^{2}=t I+\lambda A+\mu(J-I-A)
\end{aligned}
$$

where $I$ and $J$ are the identity matrix and the matrix of ones, respectively, both of order $v$. By Duval's [2] denotion, the parameters $(v, k, t, \lambda, \mu)$ of $G$ denote that $G$ is a directed graph on $v$ vertices, such that every vertex has in-degree and out-degree $k$, the number of paths of length two from a vertices $x$ to $y$ is $t$ if $x=y$, and the number of directed paths of length two directed from a vertex $x$ to another vertex $y$ is $\lambda$ if there is an arc from $x$ to $y$, and $\mu$ if there is no arc from $x$ to $y$.

\section{COUNTEREXAMPLE}

The following counterexamples have their adjacency matrices given in [1]. Consider the adjacency matrix given by a tuple

- $(v, k, t, \lambda, \mu)=(24,15,11,10,8)$, which has eigenvalues $15,3,-1$ with multiplicities $1,2,21$, respectively, elementary divisors

$$
[1,1,1,1,1,1,1,1,1,1,1,1,1,1,1,1,1,1,1,1,1,1,3,45]
$$

and determinant $-135=-3^{3} \times 5$. We see that $3^{j}$ divides $-3^{3} \times 5$ for $0 \leq j \leq 3$;

- $(v, k, t, \lambda, \mu)=(24,9,7,2,4)$, which has eigenvalues $9,1,-3$ with multiplicities $1,15,8$, respectively, elementary divisors

$$
[1,1,1,1,1,1,1,1,1,1,1,1,1,1,1,1,3,3,3,3,3,3,3,27]
$$

and determinant $59049=3^{10}$. We see that $3^{j}$ divides $3^{10}$ for $0 \leq j \leq 10$;

- $(v, k, t, \lambda, \mu)=(18,8,5,4,3)$, which has eigenvalues $8,2,-1$ with multiplicities $1,3,14$, respectively, elementary divisors

$$
[1,1,1,1,1,1,1,1,1,1,1,1,1,1,1,2,2,16]
$$

and determinant $64=2^{6}$. We see that $2^{j}$ divides $2^{6}$ for $0 \leq j \leq 6$. 
We have many possible choices for $p^{j}$ such that $p^{j} \operatorname{divides} \operatorname{det}(A) \neq 0$ which shouts out clearly that Conjecture 1 is false. The above points are some counterexamples to Conjecture 1. In Section 4, a modified statement of Conjecture 1 is given as Theorem 1 .

\section{THE RANK OF $A$ USING DETERMINANT FACTORIZATION}

In this section, we modify Conjecture 1 and prove it in some lemmas. The main result is Theorem 1.

Theorem 1. For $k, r, s$ eigenvalues of the adjacency matrix, $A$, of a directed strongly regular graph, if $k r s$ is square free and there exists $p^{j}$ such that $p^{j} \mid \operatorname{det}(A) \neq 0$ exactly, then the rank of $A$ over $\mathbb{F}_{p}$ is $v-j$, where $v$ is the order of $A$.

Lemma 1. The smallest invariant factor in the Smith normal form of $A$ is 1 . Equivalently, one of the eigenvalues $k, r$ or $s$ is \pm 1 .

Proof. The adjacency matrix has only zeros and ones in its first row, just as any other row. By the algorithm for the Smith normal form, we can have a 1 in the position $A_{(1,1)}$ and 0 in each of the positions $A_{(1, i)}, 2 \leq i \leq v$. The result follows that the resulting Smith normal form of $A$ will have a 1 among its invariant factors.

Lemma 2. The determinant of $A$ is plus or minus a product of powers of exactly two distinct primes.

Proof. By Lemma 1, we have that one of $k, r$ or $s$ is \pm 1 and that all three eigenvalues are nonzero. Since $\operatorname{det}(A)$ is the product of powers of the eigenvalues, it follows that $\operatorname{det}(A)$ is plus or minus the product of the powers of exactly two distinct primes.

Lemma 3. Let $\operatorname{det}(A)= \pm q_{1}^{\delta} q_{2}^{\psi}$ with $q_{1}, q_{2}$ distinct primes and $\delta$ and $\psi$ positive integers. For $p$ prime, if $p^{j} \mid \operatorname{det}(A)$ exactly, then $j=\delta$ or $j=\psi$.

Proof. If $p^{j} \mid \operatorname{det}(A)$ exactly, then $p^{j}=q_{1}^{\delta}$ or $p^{j}=q_{2}^{\psi}$. Since $p, q_{1}$ and $q_{2}$ are all prime numbers, the result follows.

Now we give a proof of Theorem 1 below.

Proof. By Lemmas 1, 2 and 3, if $\operatorname{det}(A) \neq 0$, then $\operatorname{det}(A)= \pm q_{1}^{\delta} q_{2}^{\psi}$ with $q_{1}, q_{2}$ distinct primes and $\delta$ and $\psi$ positive integers. The conditions that one of $k, r$ or $s$ is \pm 1 , by 
Lemma 1, and that $k r s$ is square free implies $q_{1}$ and $q_{2}$ being distinct since $k r s$ is the greatest invariant factor. If $p^{j} \mid \operatorname{det}(A) \neq 0$ exactly, then

$$
\frac{\operatorname{det}(A) \neq 0}{p^{j}}= \begin{cases} \pm q_{2}^{\psi} & \text { if } p^{j}=q_{1}^{\delta}, \\ \pm q_{1}^{\delta} & \text { if } p^{j}=q_{2}^{\psi} .\end{cases}
$$

By definition, $p$-rank of $A$ is the number of the invariant factors that are not divisible by $p$ in the Smith normal form of $A$. Since $\operatorname{det}(A) \neq 0$, then $\operatorname{rank} A=v$, and the $p$-rank of $A$ is $v-\delta$ if $p^{j}=q_{1}^{\delta}$ or $v-\psi$ if $p^{j}=q_{2}^{\psi}$. By Lemma $3, p^{j}=q_{1}^{\delta} \Longleftrightarrow j=\delta$ and $p^{j}=q_{2}^{\psi} \Longleftrightarrow j=\psi$. Therefore, the $p$-rank of $A$ is $v-j$.

\section{CONCLUSION}

The rank over a finite field of the adjacency matrix $A$ of a directed strongly regular graph was studied using the determinant bounds. It is easy to compute the spectrum of $A$ using the formulas given in [3]. We provide counterexamples to a hasty conjecture, modify the conjecture and prove the modified one. It is not clear what the rank over a finite field of the adjacency matrix $A$ would be if the largest invariant factor in the Smith normal form of $A, k r s$, is not square-free. It will be interesting to establish a result for when $k r s$ is not square-free and in general, construct a link between the $p$-rank and the decoding properties of the codes.

Acknowledgement: The author acknowledge and appreciate a suggestion on the main theorem by Pal Hegedus.

\section{REFERENCES}

[1] A. Alahmadi, A. Alkenani, J.-L. Kim, M. Shi, and P. Solé, "Directed strongly regular graphs and their codes," Bull. Korean Math. Soc, vol. 54, no. 2, pp. 497-505, 2017.

[2] A. M. Duval, "A directed graph version of strongly regular graphs," Journal of Combinatorial Theory, Series A, vol. 47, no. 1, pp. 71-100, 1988.

[3] C. D. Godsil, S. A. Hobart, and W. J. Martin, "Representations of directed strongly regular graphs," European Journal of Combinatorics, vol. 28, no. 7, pp. 1980-1993, 2007. 